\title{
DETECTING THE SOURCES OF ICE BLOCK FALLS AT THE MARTIAN NORTH POLAR SCARPS BY ANALYSIS OF MULTI-TEMPORAL HIRISE IMAGERY
}

\author{
S. Su ${ }^{1, *}$, L. Fanara ${ }^{1,2}$, X. Zhang ${ }^{3}$, K. Gwinner ${ }^{2}$, E. Hauber ${ }^{2}$, J. Oberst ${ }^{1,2}$ \\ ${ }^{1}$ Institute of Geodesy and Geoinformation Science, Technical University of Berlin, Berlin, Germany \\ - shu.su@campus.tu-berlin.de, juergen.oberst@tu-berlin.de \\ ${ }^{2}$ Institute of Planetary Research, DLR, Berlin, Germany - (Lida.Fanara, Klaus.Gwinner, Ernst.Hauber)@dlr.de \\ ${ }^{3}$ College of Surveying and Geoinformatics, Tongji University, Shanghai, China - chzhangxin@ tongji.edu.cn
}

Commission III, ICWG III/II

KEY WORDS: Mars, Polar Scarps, Block Falls, HiRISE, Change Detection.

\begin{abstract}
:
We have developed a method for automatically detecting the sources of ice block falls at the Martian north polar scarps. Multitemporal red-filter High Resolution Imaging Science Experiment (HiRISE) images were processed by using the open source NASA Ames Stereo Pipeline in combination with the USGS Integrated Software for Imagers and Spectrometers to produce $0.25 \mathrm{~m}$ resolution images as well as a $1 \mathrm{~m}$ resolution DTM. The multi-temporal HiRISE images were firstly ortho-rectified by the DTM, and then co-registered by using the Enhanced Correlation Coefficient Maximization (ECC) algorithm. We applied the change detection method on the well-aligned sub-meter scale HiRISE images, which were taken in Mars Year 29 and Mars Year 30, to investigate mass wasting at the scarp area centred at $85.0^{\circ} \mathrm{N}, 151.5^{\circ} \mathrm{E}$. The idea of the change detection method is to identify changing shadow patterns based on the grayscale difference between the images. The final results show that erosion events occurred at the full length of this study's scarp within one Mars Year. However, only the upper and lower part of the scarp show erosion activity, whereas the intermediate parts seem inactive, and this correlates with the slope.
\end{abstract}

\section{INTRODUCTION}

Martian polar regions reveal important evidence of planetary climate changes driven by changes in Mars' obliquity and eccentricity (Ward and Rudy, 1991; Byrne and Murray, 2002). From radar measurements it has been estimated that since the end of the last ice age, the polar regions have accumulated nearly 87,000 cubic kilometres of ice (Smith et al., 2016). At the same time, evidence of mass wasting, flow and debris accumulation has also been discovered (Herkenhoff et al., 2007; Hansen et al., 2011; Tesson et al., 2020).

Equatorward-facing steep scarps at the periphery of the North Polar Layered Deposits (NPLD) are known to experience various types of mass wasting, such as $\mathrm{CO}_{2}$ ice sublimation, avalanche events, and ice block falls (Ivanov and Muhleman, 2000; Russell et al., 2008; Fanara et al., 2020b). Thermoelastic modelling indicates that greater extensional stresses in winter could cause fracturing (Byrne et al., 2017). When the seasonal $\mathrm{CO}_{2}$ ice sublimates in spring, the force inside the ice layer is revealed in the form of surface fracturing. This leads to ice block falls that are playing a major role in active erosion.

Russell et al. (2007) have found abundant debris fans accumulating at the Basal Unit (BU) outcrop due to mass wasting and flow processes. Fanara et al. (2020a,b) have detected large numbers of recently fallen ice blocks at the foot of scarps by using machine learning and estimated a scarp retreat rate of $\sim 0.2$ $\mathrm{m} / \mathrm{kyr}$. Herkenhoff et al. (2020) have also obtained the volume of ice block falls through manually detecting newly appeared blocks at the foot of the scarps and found a similar rate as Fanara et al. (2020b), while Martynchuk et al. (2021) is using deep

\footnotetext{
* Corresponding author
}

learning to extend the work of Fanara et al. (2020b) and map all ice block falls of the whole north polar region through time.

However, when ice blocks detach from the steep scarps, they may break into fine material, which is not large enough to be visible in satellite images, so detailed records of the amount of mass wasting are not yet complete. A better understanding of the geological history of polar layered deposits is needed to reveal their complex climate record. Therefore, in this paper we propose exploring the sources of these block falls to investigate mass wasting. By combining the studies of ice block source areas with the identification of fresh ice blocks themselves, we can fully grasp the dynamic process of the ice block falls and conduct a seasonality analysis of ice activity at the scarp. We benefit from low-sun conditions in Mars' polar areas, where the surface morphology is revealed through cast shadows. Specifically, we search for changes in shadow patterns by using an automatic change detection method on multi-temporal images.

\section{STUDY AREA AND DATA}

Mars Reconnaissance Orbiter (MRO) High Resolution Imaging Science Experiment (HiRISE) images with scales of up to $\sim 0.25 \mathrm{~m} / \mathrm{pixel}$ have the potential to show distinguishable scarp landforms and help identify small-scale changes (McEwen et al., 2007). The study scarp with obvious fracturing features is about 22 kilometres long, centred at $85.0^{\circ} \mathrm{N}, 151.5^{\circ} \mathrm{E}$, and with 16 northern summertime HiRISE images covering it from Mars Year 29 to Mars Year 34. Figure 1 is one HiRISE image showing part of this scarp (the diagonal bright part) with a length of $\sim 7.5 \mathrm{~km}$. The scarp has a steep slope up to $70^{\circ}$. The red arrow points to the direction in which the slope descends. Below the steep NPLD scarp is the sandier Basal Unit. 


\begin{tabular}{ccccc}
\hline Image name & Resolution & Date & Ls & Usage \\
\hline PSP_009648_2650_RED & $0.25 \mathrm{~m}$ & $\begin{array}{c}2008-08-17 \\
\text { (MY29) }\end{array}$ & $113.86^{\circ}$ & Before image \\
ESP_016650_2650_RED & $0.25 \mathrm{~m}$ & $\begin{array}{c}2010-02-13 \\
(\mathrm{MY30})\end{array}$ & $51.31^{\circ}$ & Stereo Image 1 \\
ESP_016716_2650_RED & $0.25 \mathrm{~m}$ & $\begin{array}{c}2010-02-19 \\
(\mathrm{MY30})\end{array}$ & $53.58^{\circ}$ & Stereo Image 2 \\
ESP_018905_2650_RED & 0.25m & $\begin{array}{c}2010-08-08 \\
\text { (MY30) }\end{array}$ & $130.02^{\circ}$ & After image \\
\hline
\end{tabular}

Table 1. The research data list. MY means Mars Year. Ls is the solar longitude.

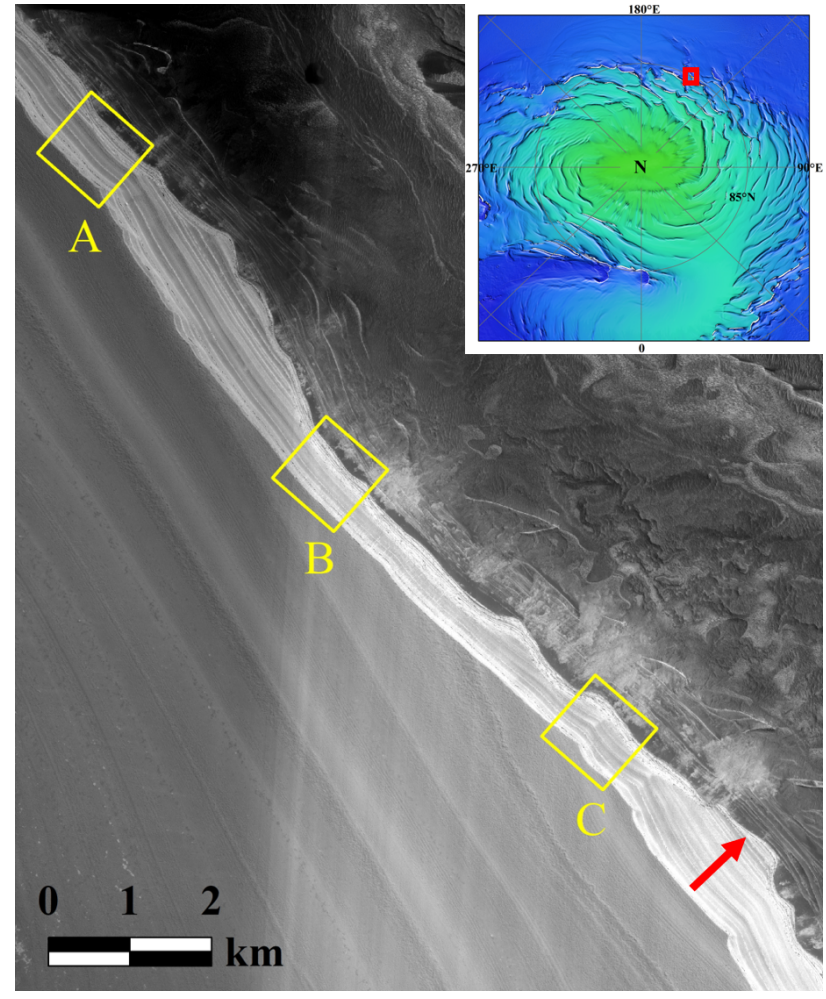

Figure 1. Red-filter HiRISE image ESP_018905_2650 at $85.0^{\circ} \mathrm{N}, 151.5^{\circ} \mathrm{E}$. The red arrow points to the direction in which the slope descends. Three yellow boxes A, B, and C are the validation areas. The location of our study area is marked as a red box on the top right map, which is the Mars MGS MOLA

Global Color Shaded Relief (Credit: USGS/NASA).

HiRISE operates on a nearly sun-synchronous orbit, which means the images are taken at the same local time of day, providing good conditions for automatically detecting changes in shadow patterns of the ice-fragments. For the change detection, we used imagery taken during the northern summertime, so that the scarps were free of the seasonal $\mathrm{CO}_{2}$ ice that covers them during the winter. For computing the Digital Terrain Model (DTM), we chose a stereo pair with similar acquisition time and suitable stereo angle. Table 1 shows the data we used in this study.

\section{3. METHODS AND EXPERIMENTS}

\subsection{Pre-processing}

The pre-processing requires a very careful ortho-rectification and co-registration to avoid artefacts in change detection. The open-source NASA Ames Stereo Pipeline (ASP) (Beyer et al., 2018) in combination with the USGS Integrated Software for Imagers and Spectrometers (ISIS) are the auxiliary tools.

A high-quality Digital Terrain Model (DTM) is the key to orthorectify HiRISE imagery. We produced a DTM with $1 \mathrm{~m}$ resolution by a series of processes on ASP. The flowchart of producing the HiRISE DTM is shown in Figure 2. All the processing steps refer to the Ames Stereo Pipeline Documentation.

Then, we used this $1 \mathrm{~m}$ DTM to ortho-rectify the "Before" HiRISE image and the "After" HiRISE image (see the list in Table 1). In most cases, the HiRISE images were not sufficiently aligned even after ortho-rectification. We performed a fineregistration of the images in two steps. First, the normalized cross-correlation was applied to find the approximate corresponding relative position of the two images. Then, the images were split into small tiles and co-registered separately by using the Enhanced Correlation Coefficient Maximization (ECC) algorithm, which is robust against photometric distortions in contrast and brightness, to provide shift and rotation correction parameters (Evangelidis and Psarakis, 2008).

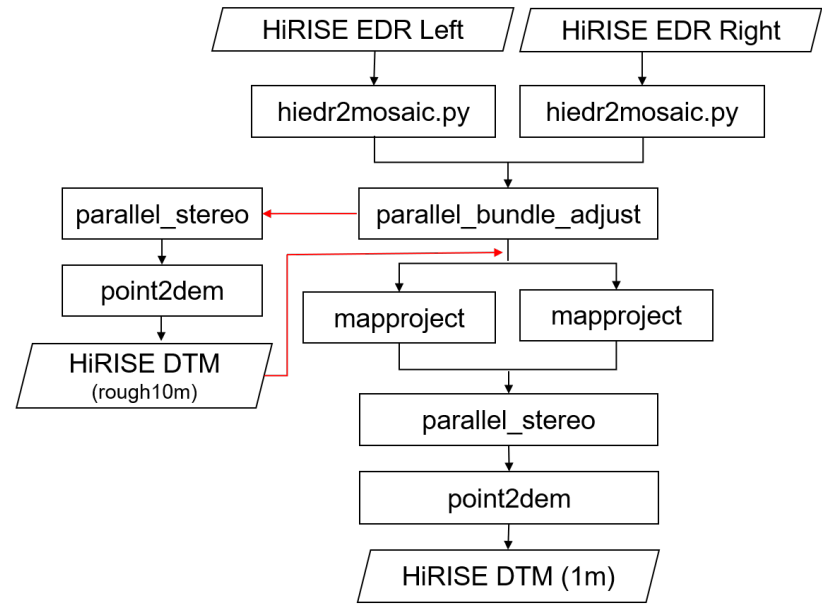

Figure 2. The Flowchart of computing the HiRISE DTM on ASP.

\subsection{Change Detection}

We benefit from the low-sun conditions in Mars' polar areas, where the surface morphology is revealed through cast shadows. The change detection is performed by shadow detection among the fractured ice-fragments. Specifically, the icefragments have shadows, depending on their sizes and sunlight incidence. When the ice-fragments fall, two phenomena may 
occur, that is, either the original shadows of the ice-fragments disappear or their surrounding parts cast new shadows. Here, we call these kinds of changes "areas of modified shadows". By comparing the relative grayscale difference between the same location of the two images, the areas of modified shadows can be finally detected.

First of all, the multi-temporal images were normalized radiometrically using the Wallis Filter (Zhang et al., 1999). The Wallis Filter adjusts the brightness values of the original image based on the mean and standard deviation of the reference image. The formula is given by:

$$
f_{(x, y)}^{\prime}=\left[f_{(x, y)}-m_{f}\right] \frac{s_{r}}{s_{f}}+m_{r}
$$

Where, $f_{(x, y)}$ is the intensity value of the original image at $(x, y)$ and $f_{(x, y)}^{\prime}$ is corresponding corrected value; $m_{f}$ and $s_{f}$ are the mean value and the standard deviation of the original image, respectively; $m_{r}$ and $s_{r}$ are the mean value and the standard deviation of the reference image, respectively.

Then, we subtracted the "Before" image from the corrected "After" image and took the absolute value of the difference. Basically, any nonzero values of the difference image are flagged as changes. However, due to the noises or radiance difference of the images, the values below the threshold can be ignored. We obtained a binary difference image by setting an empirical threshold of 50:

$$
\text { Binary Image }= \begin{cases}0, & \text { difference }<50 \\ 255, & \text { difference } \geq 50\end{cases}
$$

The pixels corresponding to 255 on the binary image are those areas, where change is suspected (the yellow, blue and red marks in Figure 3c).

However, we encountered two kinds of false detections due to specific issues. One is that the remnant white seasonal $\mathrm{CO}_{2}$ ice mimics shadow patterns. As can be seen from the yellow areas marked in figure $3 \mathrm{c}$, the remnant $\mathrm{CO}_{2}$ ice covers some parts of the scarp in the "After" image, while in the "Before" image that scarp part is free of $\mathrm{CO}_{2}$ ice. So, the grayscale difference between the two images at these parts could be higher than the threshold of 50 (Equation (1)). Our method removed such false detection by comparing the grayscale deviation between the suspected change area and its surrounding background.

The other issue is linked to imaging and illumination conditions of the multi-temporal images, which may cause discrepancies in the same shadow in the "Before" and "After" image. As can be seen from the blue area marked in figure $3 \mathrm{c}$, scale, translation or rotation of the shadows can be detected as suspected changes.

The principle of misdetection elimination that we used is to perform transformation-invariant similarity comparison on shadows. Shadow detection is a fundamental process. Here, we applied the Adaptive Gaussian Thresholding to detect the shadow areas due to the obvious grayscale differences between the shadow and its background. Adaptive thresholding determines the threshold for the pixel based on its local neighbourhood. Thus, it can handle the problem such as different lighting conditions in different areas. The formula is given by:

$$
d_{s t} t_{(x, y)}= \begin{cases}0, & \operatorname{src}_{(x, y)}>T_{(x, y)} \\ 255, & \operatorname{src}_{(x, y)} \leq T_{(x, y)}\end{cases}
$$

Where, if the pixel value at $(x, y)$ of the source image is less than threshold $T_{(x y)}$, then it is deemed as shadow pixel. In the binary result image $d s t_{(x, y)}$, pixels with value of 255 are the detected shadows. The threshold value $T_{(x y)}$ is a weighted mean of the $\mathrm{N} \times \mathrm{N}$ neighbourhood of $(x, y)$ minus a constant. For our study area the suitable parameters are $25 \times 25$ neighbourhood with the constant 10 .

After we obtained the shadows of both the "Before" and "After" images, we calculated the similarity of the corresponding shadows by using the Hu Moments, which is invariant under scale, translation and rotation $(\mathrm{Hu}, 1962)$. Image moments are often used to characterize the shape of an object in an image, while $\mathrm{Hu}$ Moments are a set of seven moment invariants calculated by using central moments. The seven moments are calculated as following:

$$
\begin{aligned}
h_{0}= & \eta_{20}+\eta_{02} \\
h_{1}= & \left(\eta_{20}-\eta_{02}\right)^{2}+4 \eta_{11}^{2} \\
h_{2}= & \left(\eta_{30}-3 \eta_{12}\right)^{2}+\left(3 \eta_{21}-\eta_{03}\right)^{2} \\
h_{3}= & \left(\eta_{30}+\eta_{12}\right)^{2}+\left(\eta_{21}+\eta_{03}\right)^{2} \\
h_{4}= & \left(\eta_{30}-3 \eta_{12}\right)\left(\eta_{30}+\eta_{12}\right)\left[\left(\eta_{30}+\eta_{12}\right)^{2}\right. \\
& \left.-3\left(\eta_{21}+\eta_{03}\right)^{2}\right]+\left(3 \eta_{21}-\eta_{03}\right)\left(\eta_{21}+\eta_{03}\right) \\
& *\left[3\left(\eta_{30}+\eta_{12}\right)^{2}-\left(\eta_{21}+\eta_{03}\right)^{2}\right] \\
h_{5}= & \left(\eta_{20}-\eta_{02}\right)\left[\left(\eta_{30}+\eta_{12}\right)^{2}-\left(\eta_{21}+\eta_{03}\right)^{2}\right] \\
& +4 \eta_{11}\left(\eta_{30}+\eta_{12}\right)\left(\eta_{21}+\eta_{03}\right) \\
h_{6}= & \left(3 \eta_{21}-\eta_{03}\right)\left(\eta_{30}+\eta_{12}\right)\left[\left(\eta_{30}+\eta_{12}\right)^{2}\right. \\
& \left.-3\left(\eta_{21}+\eta_{03}\right)^{2}\right]-\left(\eta_{30}-3 \eta_{12}\right)\left(\eta_{21}+\eta_{03}\right) \\
& *\left[\left(3 \eta_{30}+\eta_{12}\right)^{2}-\left(\eta_{21}+\eta_{03}\right)^{2}\right]
\end{aligned}
$$

where

$$
\begin{aligned}
& \eta_{i j}=\frac{\mu_{i j}}{\mu_{00}^{(i+j) / 2+1}} \\
& \mu_{i j}=\sum_{x} \sum_{y}(x-\bar{x})^{i}(y-\bar{y})^{j} f(x, y) \\
& (i \text { and } j=0,1,2 \ldots)
\end{aligned}
$$

And

$$
\bar{x}=\frac{m_{10}}{m_{00}}, \bar{y}=\frac{m_{01}}{m_{00}}
$$

Image moments capture information about the shape of a blob in a binary image because they contain information about the intensity $f_{(x, y)}$, as well as the position $x$ and $y$ of the pixel. The pixel point $(\bar{x}, \bar{y})$ is the centroid of the image $f_{(x, y)}$, the centroid moments $\mu_{i j}$ are translation invariant, and the normalized centroid moments $\eta_{i j}$ are scale invariant. Based on the normalized centroid moments, the above seven moments are invariant to image transformations.

The suspected changed areas with high similarity of the corresponding shadows are eliminated. Thus, the remaining red parts shown in figure $3 \mathrm{c}$ are the real modified shadows, which indicate that ice block falls originated there.

\section{RESULTS AND VALIDATION}

The final change detection result is visualised in Figure 4, where one red dot corresponds to one detected change. In total, 440 areas of modified shadow were detected in the study scarp area. The slope map computed from the $1 \mathrm{~m}$ resolution HiRISE DTM is shown next to the result. It is interesting that only the upper and lower part of the scarp show erosion activity, whereas the 

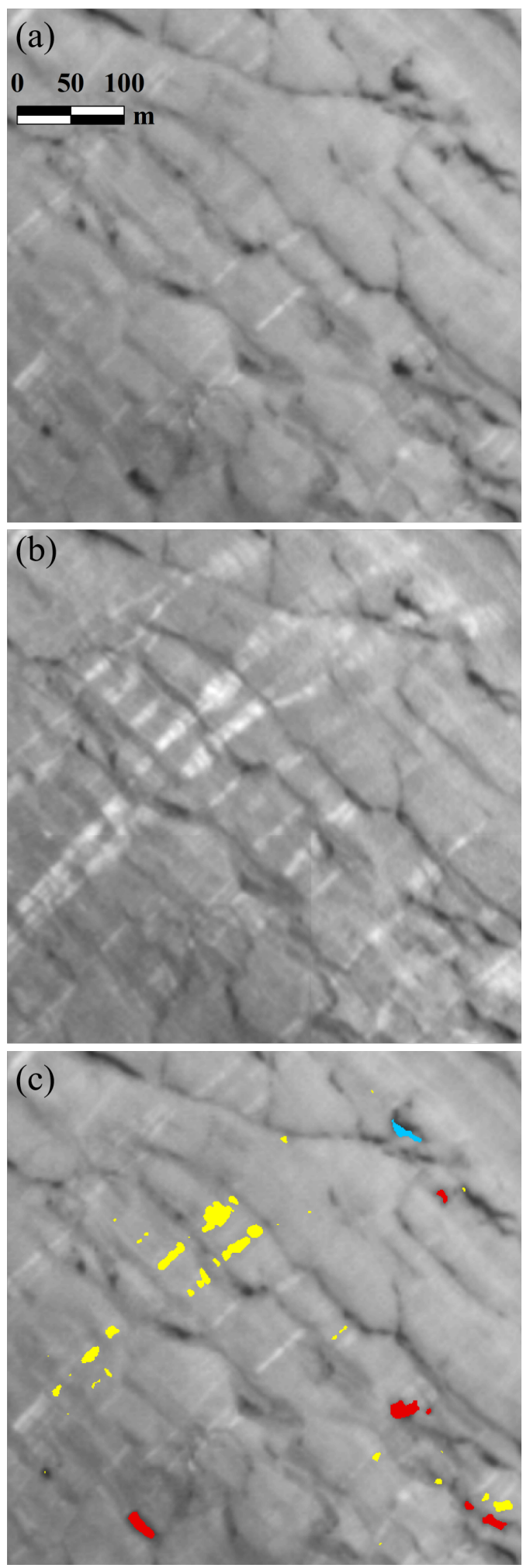

Figure 3. Detailed views of change detection process. (a) portion of the "Before" image, (b) portion of the "After" image, (c) change detection: in yellow are the detections that are automatically eliminated as false detections due to seasonal $\mathrm{CO}_{2}$ ice; in blue are the detections that are automatically eliminated as false detections caused by shadow deformation; in red are the final detections of the method.

intermediate parts seem inactive. Our results show that the mass wasting of the scarp correlates with its slope. The slopes of the two active zones shown in Figure 4 are greater than $60^{\circ}$, while the maximum slope of the middle inactive area is $40^{\circ}$.
Concerning evaluating the performance of our developed method, we chose three parts in equal distances from the left to the right of the scarp (the yellow boxes $\mathrm{A}, \mathrm{B}$ and $\mathrm{C}$ shown in Figure 2). Each part contains $4000 \times 4000$ pixels, which equals 


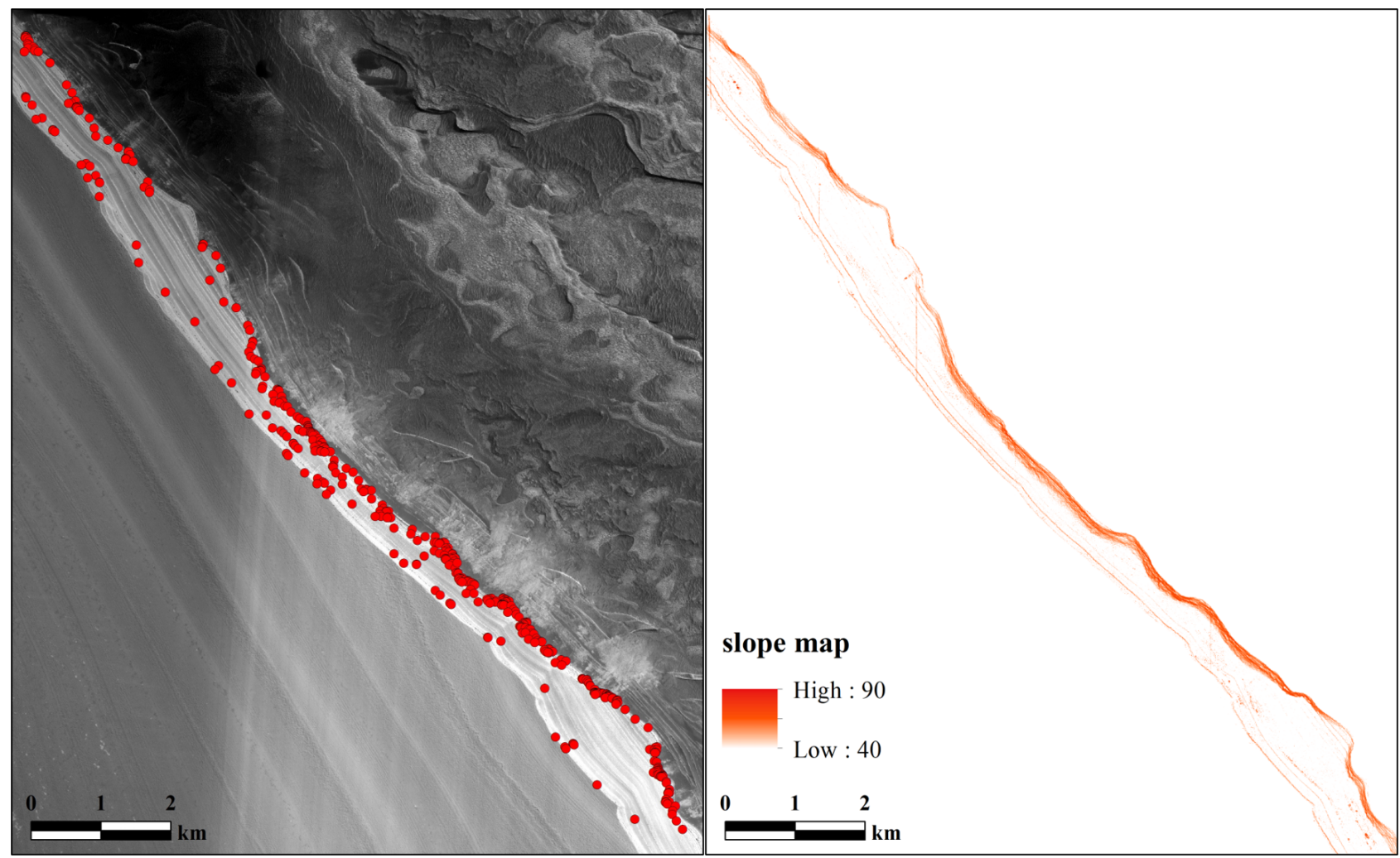

Figure 4. On the left is the change detection result (one red dot corresponds to one area of modified shadow). On the right is the corresponding slope map, only showing slopes above $40^{\circ}$.

to a $1000 \times 1000 \mathrm{~m}$ area. The results were evaluated by establishing a comparison with a manually produced map of the actual changes. We counted the number of modified shadows that were correctly detected (True Positives, TP), the number of modified shadows that were falsely detected (False Positives, FP) and the number of modified shadows that were not detected (False Negatives, FN). The evaluation is based on calculating the True Positive Rate (TPR), the False Discovery Rate (FDR), and the Quality of detection method (Q), with the following equations:

$$
\begin{aligned}
& T P R=\frac{T P}{T P+F N} \\
& F D R=\frac{F P}{T P+F P} \\
& Q=\frac{T P}{T P+F P+F N}
\end{aligned}
$$

Table 2 shows that the number of false negatives is very small, which means that our change detection method has the ability to find the modified shadows. However, there are still some falsely detected modified shadows. By checking the validation results, we attribute this to the following points:

1. Severe image deformation at the boundary area causes many false detections;

2. Differences in imaging or illumination conditions could cause shadows to shift completely, and have no overlap on the multi-temporal images. Therefore, the corresponding shadow cannot be detected, and it is retained as the changed area;

3. The intensity-based shadow detection is sensitive to image distortion, noise and local variation, which brings uncertainty to the similarity comparison.

\begin{tabular}{|c|c|c|c|}
\hline Box & TP & FP & FN \\
\hline $\mathbf{A}$ & 16 & 5 & 1 \\
\hline $\mathbf{B}$ & 27 & 6 & 1 \\
\hline $\mathbf{C}$ & 39 & 10 & 1 \\
\hline Box & TPR & FDR & $\mathbf{Q}$ \\
\hline $\mathbf{A}$ & $94.1 \%$ & $23.8 \%$ & $72.7 \%$ \\
\hline $\mathbf{B}$ & $96.4 \%$ & $18.2 \%$ & $79.4 \%$ \\
\hline $\mathbf{C}$ & $97.5 \%$ & $20.4 \%$ & $78.0 \%$ \\
\hline
\end{tabular}

Table 2. The validation results

\section{CONCLUSIONS AND OUTLOOK}

In this paper, we present our recent research on detecting morphological changes due to active erosion at the NPLD scarps through time. The proposed change detection method can perform well in detecting the sources of ice block falls at the Martian north polar scarps. The influence of seasonal $\mathrm{CO}_{2}$ ice and the difference in imaging and illumination conditions of the multi-temporal images are considered as well. We have completed the change detection for a Martian year interval (between MY29 and MY30). The results show that erosion events occurred at the full length of this scarp, and the activity of ice block falls correlates with the scarp slope. In the future, we will 
use our method to detect all sources of block falls at this scarp through time, availability of HiRISE imagery permitting. We will also probe into the flux and volume estimation for ongoing mass wasting. Our work and the research of Martynchuk et al. (2021) on block fall detection will be complementary with the common goal of monitoring the scarps and comparing the temporal and spatial variation of erosion to better understand the related driving factors.

\section{ACKNOWLEDGEMENTS}

We acknowledge the available HiRISE data, the open-source NASA Ames Stereo Pipeline (ASP) and the USGS Integrated Software for Imagers and Spectrometers (ISIS). The first author thanks China Scholarship Council (CSC) for the financial support to study in Germany.

\section{References}

Beyer, R. A., Alexandrov, O., McMichael, S., 2018. The Ames Stereo Pipeline: NASA's Open Source Software for Deriving and Processing Terrain Data. Earth Sp. Sci., 5(9), 537-548.

Byrne, S., Murray, B. C., 2002. North polar stratigraphy and the paleo-erg of Mars. J. Geophys. Res. E Planets, 107(6), 1-13.

Byrne, S., Sori, M., Russell, P., Pathare, A., Becerra, P., Molaro, J., Sutton, S., Mellon, M., Team, H., 2017. Mars polar cliffs: stressed out and falling apart. European Planetary Science Congress, 11.

Evangelidis, G. D., Psarakis, E. Z., 2008. Parametric image alignment using enhanced correlation coefficient maximization. IEEE Trans. Pattern Anal. Mach. Intell., 30(10), 18581865.

Fanara, L., Gwinner, K., Hauber, E., Oberst, J., 2020a. Automated detection of block falls in the north polar region of Mars. Planet. Space Sci., 180(September), 104733. https://doi.org/10.1016/j.pss.2019.104733.

Fanara, L., Gwinner, K., Hauber, E., Oberst, J., 2020b. Presentday erosion rate of north polar scarps on Mars due to active mass wasting. Icarus, 342(September 2019), 113434. https://doi.org/10.1016/j.icarus.2019.113434.

Hansen, C., Bourke, M., Bridges, N. T., Byrne, S., Colon, C., Diniega, S., Dundas, C., Herkenhoff, K., McEwen, A., Mellon, M. et al., 2011. Seasonal erosion and restoration of Mars' northern polar dunes. Science, 331(6017), 575-578.

Herkenhoff, K., Byrne, S., Dundas, C., Baugh, N., Hunter, M., 2020. HiRISE Observations of Recent Phenomena in the North Polar Region of Mars. LPI Contributions, 2099, 6059.

Herkenhoff, K. E., Byrne, S., Russell, P. S., Fishbaugh, K. E., McEwen, A. S., 2007. Meter-scale morphology of the north polar region of Mars. Science, 317(5845), 1711-1715.

Hu, M.-K., 1962. Visual pattern recognition by moment invariants. IRE transactions on information theory, 8(2), 179-187.

Ivanov, A. B., Muhleman, D. O., 2000. The Role of Sublimation for the Formation of the Northern Ice Cap: Results from the Mars Orbiter Laser Altimeter. Icarus, 144(2), 436-448.
Martynchuk, O., Fanara, L., Hauber, E., Oberst, J., Gwinner, K., 2021. Computer vision model for detecting block falls at the martian north polar region. EGU General Assembly 2021, 19-30 Apr 2021, EGU21-15776.

McEwen, A. S., Eliason, E. M., Bergstrom, J. W., Bridges, N. T., Hansen, C. J., Delamere, W. A., Grant, J. A., Gulick, V. C., Herkenhoff, K. E., Keszthelyi, L., Kirk, R. L., Mellon, M. T., Squyres, S. W., Thomas, N., Weitz, C. M., 2007. Mars reconnaissance orbiter's high resolution imaging science experiment (HiRISE). J. Geophys. Res. E Planets, 112(5), 5-6.

Russell, P. S., Byrne, S., Herkenhoff, K., Fishbaugh, K., Hansen, C., Thomas, N., McEwen, A., Team, H., 2007. North polar basal stratigraphy and active mass-wasting processes from hirise with implications for polar scarp evolution. Seventh International Conference on Mars, 1353, 3377.

Russell, P., Thomas, N., Byrne, S., Herkenhoff, K., Fishbaugh, K., Bridges, N., Okubo, C., Milazzo, M., Daubar, I., Hansen, C., McEwen, A., 2008. Seasonally active frost-dust avalanches on a north polar scarp of Mars captured by HiRISE. Geophys. Res. Lett., 35(23), 4-5.

Smith, I. B., Putzig, N. E., Holt, J. W., Phillips, R. J., 2016. An ice age recorded in the polar deposits of mars. Science (80-. )., 352(6289), 1075-1078.

Tesson, P.-A., Conway, S., Mangold, N., Ciazela, J., Lewis, S., Mège, D., 2020. Evidence for thermal-stress-induced rockfalls on Mars impact crater slopes. Icarus, 342, 113503.

Ward, W. R., Rudy, D. J., 1991. Resonant obliquity of Mars? Icarus, 94(1), 160-164.

Zhang, L., Zhang, Z., Zhang, J., 1999. The image matching based on wallis filtering. Journal of Wuhan Technical University of Surveying and Mapping, 24(1), 24-27. 